\title{
Uma investigação do problema de elaboração de grade horária (timetabling problem)
}

\author{
Verçosa, L. F. V \\ Escola Politécnica de Pernambuco \\ Universidade de Pernambuco \\ 50.720-001 - Recife, Brasil \\ lfvv.eliane@dsc.upe.br
}

\author{
Loiola, E. M. \\ Escola Politécnica de Pernambuco \\ Universidade de Pernambuco \\ 50.720-001 - Recife, Brasil
}

Resumo Este trabalho apresenta o problema de elaboração de grade horária (timetabling problem) no contexto do curso de Engenharia de Computação da Universidade de Pernambuco e também a geração de uma solução viável para uma instância do problema. Para tanto, utiliza-se dos conceitos de escalonamento e coloração de grafos que são amplamente discutidos e exemplificados.

\footnotetext{
Abs- $\quad$ This work show the timetabling problem in the contexts of Computer Engineering course of the Unitract versity of Pernambuco and even generate na feasible solution for one instance of this problem. For doing that, is used scheduling concepts and graphs coloring that are largely discussed and exemplified.
} 


\section{Introdução}

A definição de escalonamento é uma descrição do ordenamento de recursos no tempo ou no espaço, tentando alcançar com sucesso um determinado objetivo e respeitando o conjunto de limitações impostas (Beasley, 1997). O problema de escalonamento está relacionado a inúmeras aplicações nas áreas de planejamento de produção, programação de horário, escala de trabalho entre outros.

O problema de elaboração de grade horária de universidades é uma variação do problema de escalonamento, mais conhecido como timetabling problem, cujo principal objetivo é alocar um conjunto de professores a um conjunto de horários e salas satisfazendo as restrições envolvidas. Tais restrições podem ter natureza impeditiva ou não impeditiva. A violação de restrições de natureza impeditiva está relacionada com soluções que são inviáveis, já a violação de restrições não impeditivas está relacionada com soluções que apesar de serem viáveis não são soluções desejadas. Uma solução desejada é aquela que satisfaz todo o conjunto de restrições associadas ao problema.

O caso geral é NP-árduo (Even et al. 1976). Na literatura existe uma variedade de técnicas propostas para resolver o timetabling problem e suas variações (Schaerf, 1999). Entre elas estão diversas metaheurísticas tais como tabu search e algoritmos genéticos (Chu and Fang, 1999; Santos et al. 2005). O timetabling problem também pode ser visto como um problema de coloração de grafos (Even et al. 1976), tornando-se uma abordagem alternativa para o problema.

Esta pesquisa foi aplicada no contexto da Universidade de Pernambuco, usando como estudo de caso a grade horária do curso de Engenharia de Computação.

No curso de Engenharia de Computação e demais cursos da Escola Politécnica da Universidade de Pernambuco (UPE) cabem aos coordenadores, de seus respectivos cursos, as tarefas de estabelecer os horários das disciplinas do curso e distribuí-las entre os professores de acordo com os horários preestabelecidos. O principal objetivo é evitar choques de horários entre professores habilitados e disponíveis, entre outras restrições. Todo esse trabalho é feito de forma manual possuindo assim as desvantagens citadas anteriormente.

\section{0 problema do Escalonamento}

Em Viana (1996) encontramos a definição matemática a seguir para o problema de escalonamento.
Dado um conjunto de $n$ de tarefas independentes com tempos de duração $t_{1}, t_{2}, \ldots, t_{n}$, onde $t i$ é o tempo de execução da tarefa $i$, e um conjunto de $m$ processadores idênticos, inicialmente ociosos, que funcionam em paralelo. Distribuir (escalonar) as $n$ tarefas pelos $m$ processadores de forma a minimizar o tempo de execução, considerando que cada tarefa será executada uma única vez em um dos processadores. Ou seja:

$$
\begin{aligned}
& \min Z \geq \sum_{i-j}^{n} t_{i} x_{i j}, j \in\{1, \ldots, m\} \\
& \sum_{j=1}^{n} x_{i j}=1, i \in\{1, \ldots n\} \quad \forall i, j, \text { e } x_{i j} \in\{0,1\}
\end{aligned}
$$

A expressão (1.2) nos mostra a restrição de que cada tarefa só é executada uma única vez em algum dos processadores unicamente. No entanto, observa-se que um processador poderá executar diversas tarefas. Já a expressão (1.1) nos mostra que deseja-se minimizar $z$, ou seja, minimizar o tempo total de execução das tarefas em cada processador. As Figuras 1 e 2 apresentam um exemplo retirado de Viana (1996), neste exemplo podemos observar uma lista de tarefas antes e após seu escalonamento.

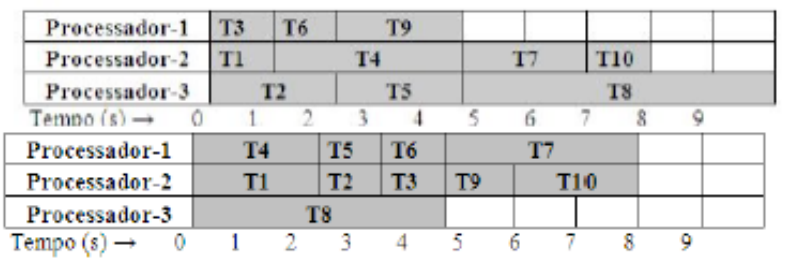

Fig 1. Distribuição de tarefas antes do escalonamento.

Fig 2. Distribuição de tarefas após escalonamento.

\section{O problema de Elaboração de Grade Horária}

O problema de elaboração de grade-horária trata-se de uma variação do problema de escalonamento, onde é preciso escalonar uma quantidade de professores (unidades de processamento) para uma quantidade de aulas (tarefas) de maneira que sejam satisfeitas certas restrições pedagógicas e administrativas. Souza et al. (2000) definem o problema e abordam um método de resolução heurístico que melhora resultados existentes na literatura e Souza et al. (2001) tratam de novos procedimentos para melhoria das soluções encontradas no trabalho anterior. O procedimento definido em Souza et al. (2000) consiste basicamente em diminuir o somatório da função objetivo de cada professor respeitando à restrições (administrativas e pedagógicas). Essa função mede essencialmente a qualidade do 
horário de cada professor levando em conta fatores de maior ou menor peso sendo dada por:

$$
f o=\sum_{i=1}^{m} f Q=\sum_{i=1}^{m} \alpha_{i}^{*} b_{i}+\beta_{i}^{*} v_{i}
$$

Onde:

$f o=$ somatório das funções objetivos dos professores;

$f_{i}^{o}=$ função objetivo do professor i;

$b_{i}=$ horário ocioso entre dois horários de aula de um mesmo professor (buracos);

$v_{i}=$ número de dias em que um professor está envolvido em alguma atividade;

$\alpha_{i} e \beta_{i}=$ pesos que refletem a importância relativa de $b_{i}$ e $v_{i}$

$m=$ quantidade de professores;

O exemplo abaixo retirado de Souza et al. (2000) nos esclarece o objetivo do procedimento proposto, veremos que não será necessário descrever as restrições para entendimento do exemplo. A Tabela 1 mostra o fragmento do quadro $Q_{i}$ de horários de professores. Cada linha $i$ representa um professor $(i=t 1, t 2, t 3, t 4)$ e cada coluna $k$ um horário $(k=h 1, h 2, h 3, h 4, h 5)$ de um dia da semana. Cada par $(i, k)$ da tabela representa a atividade do professor $i$ no horário $k$, cada uma dessas atividades equivale a um encontro $m_{i j}$ onde $i$ representa um professor e $j$ uma turma. $c 1, c 2, c 3$ e $c 4$ são turmas. Um traço (-) significa indisponibilidade do professor, enquanto uma célula vazia indica que não há atividade no horário. A coluna $f_{i}^{o}$ indica o valor da função objetivo de cada professor, expressa em (1.3), $\operatorname{com} \alpha_{i}=1$ e $\beta_{i}=0$.

\begin{tabular}{|l|l|l|l|l|l|}
\hline & $\boldsymbol{h}_{\mathbf{1}}$ & $\boldsymbol{h}_{\mathbf{2}}$ & $\boldsymbol{h}_{3}$ & $\boldsymbol{h}_{\mathbf{4}}$ & $\boldsymbol{h}_{\mathbf{5}}$ \\
\hline$t_{\boldsymbol{1}}$ & $c_{1}$ & & $c_{2}$ & $c_{2}$ & \\
\hline $\boldsymbol{t}_{2}$ & $c_{2}$ & & & $c_{1}$ & $c_{1}$ \\
\hline $\boldsymbol{t}_{\mathbf{3}}$ & & $c_{2}$ & $c_{1}$ & $c_{3}$ & $c_{2}$ \\
\hline $\boldsymbol{t}_{\mathbf{4}}$ & $c_{3}$ & $c_{1}$ & $c_{3}$ & $c_{4}$ & - \\
\hline
\end{tabular}

\begin{tabular}{|c|}
\hline$f \hat{o}_{i}$ \\
\hline 1 \\
\hline 2 \\
\hline 0 \\
\hline 0 \\
\hline
\end{tabular}

Fig 3. Quadro $Q_{1}$.

Por exemplo, a função $f o 1=1$ indica que há um intervalo vazio entre dois horários do professor $t 1$ e assim por diante. Temos então que $f\left(Q_{1}\right)=1+2+0+0=3$. Para melhorar essa função objetivo o procedimento associa um grafo para cada turma e por meio da manipulação de ciclos de custo negativo melhora a função objetivo.
Dessa forma, após a aplicação do procedimento, obtemos a Figura 4, onde destacamos as mudanças de escalonamento.

\begin{tabular}{|l|l|l|l|l|l|}
\hline & $\boldsymbol{h}_{\boldsymbol{1}}$ & $\boldsymbol{h}_{\mathbf{2}}$ & $\boldsymbol{h}_{\mathbf{3}}$ & $\boldsymbol{h}_{\boldsymbol{d}}$ & $\boldsymbol{h}_{\boldsymbol{5}}$ \\
\hline $\boldsymbol{t}_{1}$ & & & $c_{2}$ & $c_{2}$ & $c_{1}$ \\
\hline $\boldsymbol{t}_{\mathbf{2}}$ & $c_{2}$ & & $c_{1}$ & $c_{l}$ & \\
\hline $\boldsymbol{t}_{3}$ & $c_{1}$ & $c_{2}$ & & $c_{3}$ & $c_{2}$ \\
\hline $\boldsymbol{t}_{4}$ & $c_{3}$ & $c_{1}$ & $c_{3}$ & $c_{4}$ & - \\
\hline
\end{tabular}

\begin{tabular}{|c|}
\hline$f \sigma_{j}$ \\
\hline 0 \\
\hline 1 \\
\hline 1 \\
\hline 0 \\
\hline
\end{tabular}

Fig 4. Quadro $Q_{1}^{\prime}$ com melhoria da função objetivo.

A manipulação de ciclos de custo negativo possui basicamente o seguinte entendimento: a mudança no horário da aula de uma turma implica numa reação em cadeia, ocasionando outras mudanças a serem realizadas na turma de maneira que o horário liberado pela primeira modificação seja ocupado por outro professor fechando assim um ciclo. Isso pode ser observado com a mudança do horário da aula da Turma $c l$ de $h 1$ para $h 5$ que implicou na seguinte seqüência de mudanças: $(c 1, t 1) \quad h_{1} \rightarrow h_{5} \rightarrow(c 1, t 2)$

$h_{5} \rightarrow h_{3} \rightarrow(c l, t 3) \quad h_{3} \rightarrow h_{1}$. Esse mesmo procedimento é realizado para cada turma.

Outro algoritmo que consiste na transferência de um professor de uma turma para outra em um mesmo horário é encontrado em (Souza et al., 2001). Neste algoritmo, uma solução inicial é gerada por um procedimento construtivo parcialmente guloso, sendo seguido de uma busca tabu como procedimento de busca local. Quando é gerada uma solução sem sobreposições, mas com algum outro tipo de inviabilidade, aciona-se um procedimento para tentar recuperar a viabilidade. Sendo bem sucedido, ele é novamente acionado agora para tentar melhorar a agenda dos professores. Após um número de iterações sem melhorias, todo o processo (fase construtiva e busca local) é repetido até que uma condição de parada seja satisfeita. $\mathrm{O}$ algoritmo proposto em (Souza et al., 2001) é uma melhoria do algoritmo proposto em (Souza et al., 2000).

\section{Definição do Grafo do Timetabling Problem}

Existem diversos tipos de coloração para os grafos, tais como: coloração de vértices, de arestas, e total. Nosso problema aborda a coloração de vértices. A seguir serão apresentados algumas definições de grafos extraídos de Friedmann et al. (2010) importantes para essa pesquisa.

Definição 1. Coloração própria de $G$ é uma atribuição de cores aos elementos do grafo, sejam vértices, arestas ou ambos, de tal forma que elementos adjacentes recebem cores distintas. 
Definição 2. Uma $t$-coloração de $G$ é uma coloração com $t$ cores que são capazes de colorir um grafo (não necessariamente o menor conjunto de cores).

Tem-se então a definição matemática para a coloração de vértices dada a seguir.

Segundo Neufeld et al. (1974) o problema de gradehorária (professor-turma) é definido como a seguir: um conjunto de professores $T=\left\{t_{i}\right\}$ com $i=1, \ldots, \alpha$; um conjunto de turmas $C=\left\{c_{j}\right\} \operatorname{com} j=1, \ldots, \beta$; um conjunto de horários $H=\left\{H_{k}\right\}$ com $k=1, \ldots, t$. O produto cartesiano $\alpha \times \beta$ é representado pela matriz $R=\left[r_{i j}\right.$ ], onde $r_{i j} \geq 0$. $\mathrm{O}$ valor $r_{i j}$ é igual ao número de horários que o professor $t_{i}$ encontra a turma $c_{j}$. O problema de grade-horária deve satisfazer certas condições pré-estabelecidas. Essas condições são restrições de indisponibilidade e encontros préestabelecidos. Seja $n, 1 \leq n \leq r i j$, o enésimo encontro entre o professor $t_{i}$ e a turma $c_{j}$

representado por $m_{i j}^{n} . M_{i j}^{n}=\left\{M_{i j}^{1}, \ldots, M_{i j}^{r_{i j}^{n}}\right\}$ representa todos os encontros $r_{i j}$.

Seja $M_{j}=\bigcup_{i=1}^{a} r_{i j}$ então $M_{j}$ representa os encontros da turma $c_{j}$ com todos os professores $t_{i} \in T$.

Qualquer solução para o problema de grade horária descreve todos os horários $r_{i j}$ em que cada encontro $m_{i j}^{n}$ das turmas $c_{j}=1, \ldots, \beta$ com os professores $t_{i}=1, \ldots, \alpha$ devem acontecer.

Seja $G$ um grafo com um conjunto de vértices $V$ e um conjunto de arestas não direcionadas $E$. Define-se $V$ tal que seus vértices $\mathrm{v} \in \mathrm{V}$ possuem uma correspondência umpara-um com os encontros $M_{i j}^{n}$.

Assim, cada vértice será representado por $v_{i j}^{n}$ onde $i$ representa a turma, $j$ o professor e $n$ o enésimo encontro entre os dois. $v_{i j}$ corresponderá a $M_{i j}$ e $V_{j}$ corresponderá a $M_{j}$.

Define-se por fim o conjunto de arestas $E$ do grafo $G$ como $E=E^{1} \cup E^{2}$ onde $E^{1}=\cup_{j=1}^{\beta} E_{j}$, com $E_{j}=$ $\left\{\left(v, v^{\prime}\right) \mid v, v^{\prime} \in V_{j}, v \neq v^{\prime}\right\}$ e $E^{2}=\bigcup_{i=1}^{\alpha} E_{i j j}$, onde $E_{i j j}=$ $\left\{\left(v, v^{\prime}\right) \mid v \in V_{i j}, v^{\prime} \in V_{i j}\right\}$ para todo $j \neq j^{\prime} \operatorname{com} r_{i j}, r_{i j}{ }^{\prime}$ $>0$ e $E_{i j j^{\prime}}=\varnothing$ caso contrário.

Assim, nota-se que o conjunto $E^{1}$ especifica que todos os vértices de uma mesma turma, $v \in V_{j}$, estarão interconectados, assim como $E^{2}$ nos diz que todos os vértices de turmas diferentes $(j$ e $j$ '), mas que possuem o mesmo professor $i$ também serão interconectados. Para facilitar a compreensão, criamos um exemplo básico dado para os conjuntos dados a seguir:

$$
\begin{aligned}
& \mathrm{T}=\left\{t_{1}, t_{2}, t_{3}\right\} \\
& \mathrm{C}=\left\{c_{1}, c_{2}\right\} \\
& \mathrm{H}=\left\{h_{1}, h_{2}, h_{3}, h_{4}, h_{5}\right\} \\
& M_{1}=\left\{m_{11}^{1}, m_{11}^{2}, m_{21}^{1}, m_{21}^{2}, m_{31}^{1}\right\} \\
& M_{2}=\left\{m_{12}^{1}, m_{12}^{2}, m_{22}^{1}, m_{22}^{2}, m_{32}^{1}\right\}
\end{aligned}
$$

Para cada $M_{i j}^{n}$ haverá um vértice $v_{i j}^{n}$ correspondente. Desta forma, obtém-se o grafo a seguir.

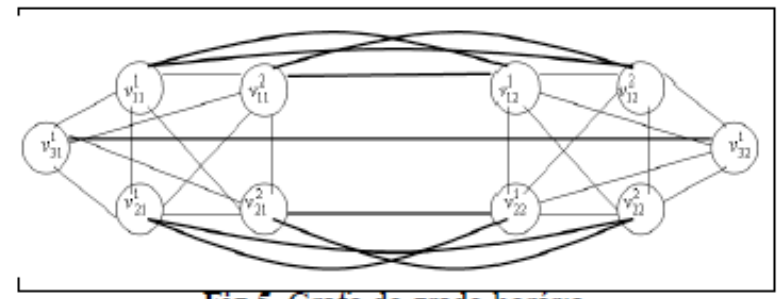

Fig 5. Grafo de grade-horárı.

A Figura 1 permite visualizar e compreender melhor o problema. As arestas do subconjunto $E^{2}$ estão em negrito para melhorar a visualização por meio da distinção entre as arestas do subconjunto $E^{1}$ e as arestas do subconjunto $E^{2}$.

\section{Coloração do Grafo do Timetabling}

Para efetuar a coloração, as restrições são gradativamente inseridas. Primeiro se estabelece uma relação do problema de coloração com um problema de grade-horária sem qualquer tipo de restrição.

Seja $L=l_{1}, l_{2}, \ldots, l$ suponha que os horários $h_{1}$, $h_{2}, \ldots, h_{t} \in H$ correspondem respectivamente às cores $=$ $l_{1}, l_{2}, \ldots, l_{t}$. A atribuição do horário $h_{k}$ a um encontro $m_{i j}^{n}$ corresponde a atribuição da cor $l_{k}$ para o vértice $v_{i j}^{n}$.

Uma vez que qualquer solução para o problema de grade-horária descreve todos os horários $r_{i j}$ em que cada encontro $m_{i j}^{n}$ das turmas $c_{j}=1, \ldots, \beta$ com os professores $t_{i}=1, \ldots, \alpha$ devem acontecer, então a correspondência estabelecida cria uma relação um-para-um entre todas as soluções possíveis para o problema e todas as $t$ - olorações possíveis para o grafo correspondente.

Por fim são adicionadas as restrições de indisponibilidade e de encontros pré-estabelecidos. Uma vez que $h_{k}$ se relaciona com a $l_{k}$ cor, dizer que um professor $x$ está indisponível em um horário $h_{k}$ equivale a dizer que todos os vértices do tipo $v_{x j}^{n}$ não poderão receber a cor $l_{k}$. A mesma linha de raciocínio é válida para uma turma $y$ indisponível em um horário $h_{k}$. Já, dizer que um professor $z$ terá um 
encontro $n$ pré-estabelecido com uma turma w em um horário $h_{k}$, significa dizer que o vértice correspondente $v_{z j}^{n}$ será obrigado a receber de antemão a cor $l_{k}$. Esta restrição está presente no estudo de caso do projeto, ou seja, na grade horária do curso de Engenharia de Computação da POLI, já que os horários dos professores do ciclo básico são pré-estabelecidos.

Para facilitar a assimilação desses conceitos apresentamos o grafo do exemplo anterior acrescentando-se as seguintes restrições: professor $t 3$ está indisponível em $h 1 \mathrm{e}$ $h 2$; professor $t 1$ precisa encontrar turma $c 2$ em $h 3$ e $h 4$. Iremos utilizar as seguintes cores:

$$
\begin{gathered}
h_{1} \rightarrow l_{1}=\text { vermelho } \\
h_{2} \rightarrow l_{2}=\text { azul claro } \\
h_{3} \rightarrow l_{3}=\text { amarelo } \\
h_{4} \rightarrow l_{4}=\text { verde } \\
h_{5} \rightarrow l_{5}=\text { rosa }
\end{gathered}
$$

Desta forma, temos uma solução viável para coloração do grafo com as restrições dadas:

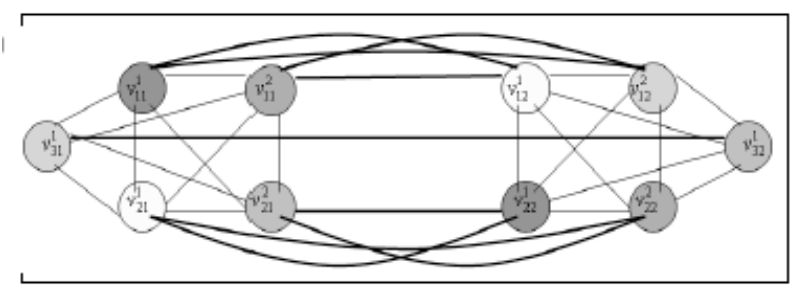

Fig 6. Solução viável para o problema de grade horária.

Observa-se que uma solução viável para o problema pode não ser uma solução ótima.

\section{Algoritmo de Coloração}

A formulação por grafo para o problema permite atender os requisitos a seguir:

1. Nenhum professor poderá ministrar aulas em mais de uma turma ao mesmo tempo;

2. Nenhuma turma poderá ter aula com mais de um professor ao mesmo tempo;

3. Os professores poderão ministrar aulas somente em seus horários disponíveis;

4. As turmas poderão ter aulas do ciclo profissional somente em horários que não estiverem reservados às disciplinas do ciclo básico.
Assim, o algoritmo de coloração, baseado em Silva et al. (2010), consultará o grafo para satisfazer as restrições 1 e 2 , e criará as restrições 3 e 4 .

São parâmetros de entrada do algoritmo: o grafo $G$ e um vértice $s$, o primeiro a ser colorido. O conjunto $Y$ representa o conjunto de vértices não coloridos. O parâmetro horário-cor corresponde (no final da execução do algoritmo) ao resultado da coloração e representa um vetor que possui em cada posição um conjunto de vértices (cada vértice é um encontro $n$ entre um professor e uma turma) os quais receberão a mesma cor (horário).

O algoritmo é mostrado pelo pseudocódigo abaixo, cujos passos são repetidos enquanto houver vértices não coloridos no grafo:

Passo 1. Definir uma nova posição no vetor Horário[cor] (novo horário);

Passo 2. Adicionar em Horário[cor], dentre os vértices não coloridos, aqueles que atendem às restrições do problema;

Passo 3. Incrementar o número de cores.

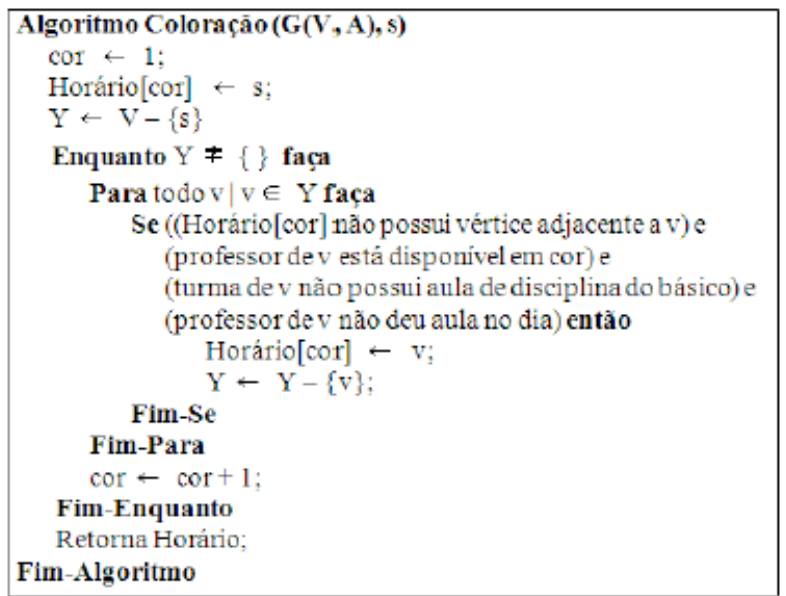

Fig 7. Algoritmo de Coloração do Grafo do Timetabling Problem.

A condição "Se Horário[cor] não possui adjacente" verifica através do grafo se as condições 1 e 2 são satisfeitas. A condição "Se professor está disponível em cor" verifica a condição 3 e por último, a condição "Se turma não possui aula de disciplina do básico" verifica a condição 4. Finalmente a condição "Se professor de $v$ não deu aula no dia" impede que um mesmo professor e uma mesma turma possam se encontrar duas vezes no mesmo dia (cada encontro tem duração de uma hora e cinqüenta minutos). 


\section{Estudo de Caso do Curso de Enge- nharia de Computação}

Na Escola Politécnica de Pernambuco (núcleo das engenharias da Universidade de Pernambuco), o vice- coordenador Dr. Sérgio Campello é atualmente o responsável por elaborar semestralmente a gradehorária de todas as turmas e professores do curso de Engenharia de Computação. Em entrevista com ele foi possível coletar importantes informações a respeito de como é feito todo o processo. Primeiro, é necessário dois a três dias para montar manualmente todos os horários e em seguida mais três ou quatro dias para fazer mudanças de acordo com as necessidades e preferências dos professores. Assim, temos abaixo uma descrição passo-a-passo de todo o processo:

$1^{\circ}$ PASSO: Distribuir todas as disciplinas disponíveis para todos os docentes. Nessa distribuição a quantidade de atribuições (administrativas, acadêmicas, etc.) de cada docente e sua disponibilidade são levados em conta para saber quantas disciplinas o professor deverá assumir. Estes fatores podem ser, por exemplo: cargo administrativo (como coordenador, por exemplo), orientação de TCC, aulas no mestrado, etc.

$2^{\mathbf{0}}$ PASSO: Para cumprir com o passo acima, um questionário é enviado para o time de docentes. Neste questionário, o docente poderá informar suas disciplinas preferidas e horários disponíveis. Na primeira tabela o docente deverá informar suas restrições de horário e na segunda, suas preferências de horário.

$3^{\circ}$ PASSO: Após a coleta dos dados, o horário de cada turma e de cada docente é elaborado manualmente. Sendo necessário considerar que os horários das disciplinas do ciclo básico são fixos e não podem sofrer qualquer alteração. Sendo assim, cada horário é elaborado buscando sempre otimizar de modo a atender as necessidades dos professores, ou seja, tentando reduzir a quantidade de dias de trabalho e os "buracos" existentes entre uma aula e outra de cada professor. Minimizar a quantidade de dias de trabalho dos professores é uma das maiores dificuldades, assim como evitar choques de disciplinas para alunos que tenham sido reprovados. Além disso, é preciso levar em conta a quantidade de feriados no semestre em questão. Por exemplo, se em um semestre há muitos feriados na terça, então muitas segundas serão imprensadas, consequentemente, deve-se evitar a alocação de uma mesma disciplina nestes dois dias, caso contrário será difícil repor as aulas perdidas e cumprir a carga horária semestral para a mesma.

$4^{\circ}$ PASSO: Se necessário o Vice-Coordenador ainda poderá fazer modificações nos horários de turmas e professores de maneira a satisfazer as "restrições de última hora" (não especificadas nos questionários dos professores) e restrições de professores que porventura não preencheram o questionário (nesse caso dar-se-á preferência aos que preencheram o questionário).

\section{Resultados}

Foram usados a linguagem Java e o ambiente eclipse para implementar os algoritmos e gerar a solução. $O$ estudo de caso foi feito com as seguintes variáveis:

- Utilizou-se a grade-horária elaborada manualmente em 2010.2 para saber as disciplinas lecionadas por cada um dos 19 professores do ciclo profissional.

- Estabeleceu-se que existem apenas três horários para execução de aulas por dia (segunda a sexta): $7: 10 \mathrm{~h}$ às $8: 50 \mathrm{~h}, 8: 50 \mathrm{~h}$ às $10: 30 \mathrm{~h}$ e $10: 30 \mathrm{~h}$ às 12:00h.

- Utilizou-se o conjunto de 10 turmas onde cada uma se encontra em um período diferente.

- Não foi incluído no estudo de caso disciplinas lecionadas fora desse conjunto de horários.

Para geração dos resultados foram seguidos os seguintes passos:

Passo 1. Geração do grafo descrito em 3

Passo 2. Coloração do grafo através do algoritmo descrito em 4.1

No Passo 1 são usados três arquivos no formato txt para gerar o grafo definido em Koffman etal. (2008). O arquivo ProfessorHorário especifica os horários indisponíveis dos professores, ProfessorTurma, a quantidade de aulas entre cada par professor-turma e RestriçãoHorárioTurma, os horários das disciplinas do ciclo básico. Assim como pode ser visualizado na Figura 8, o arquivo ProfessorTurma corresponde a uma matriz binária $A=[a i j]$ em que o valor aij $=1$ significa que o professor $j$ está disponível (não tem compromisso pessoal impeditivo) para lecionar no horário $i$. Analogamente, se $a i j=0$ então professor $j$ estará indisponível. A partir desses arquivos o programa gera internamente o grafo. 


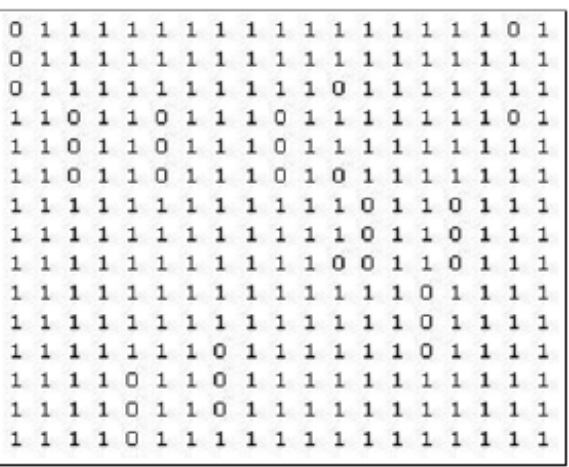

Fig 8. Arquivo ProfessorHorário, colunas representam professores ( 0 a 18) e linhas representam horários ( 0 a 15).

Para facilitar a leitura, a Figura 9 mostra o nomes dos professores correspondentes às colunas da matriz ProfessorHorário. A Figura 10 mostra a matriz $B=[b i j]$ do arquivo ProfessorTurma, onde o valor bij mostra a quantidade de aulas do professor $j$ para a turma $i$. Por fim, a Figura 11 mostra o arquivo RestriçãoHorárioTurma onde cada linha possui os horários indisponíveis das turmas de cada período. O caracter '-' indica que a turma da linha correspondente não possui horários indisponíveis. Com essas instâncias foi gerado o grafo do Passo 1. Para execução do Passo 2 escolheu-se primeiramente um vértice inicial de maneira que resulta-se em menor função objetivo (descrita em 3.2). Após isso foram gerados os resultados mostrados nas Figuras 12 e 13.

\begin{tabular}{|ll|}
\hline 0 & Bruno Fernandes \\
1 & Byron Leite \\
2 & Carmelo \\
3 & Daniel Chaves \\
4 & Edison \\
5 & Eliane \\
6 & Fernando Buarque \\
7 & Genésio \\
8 & Gustavo Carvalho \\
9 & Simone Santos \\
10 & José Paulo \\
11 & Luis Nenezes \\
12 & Haria Lencastre \\
10 & wamar
\end{tabular}

Fig 9. Relação de professores.

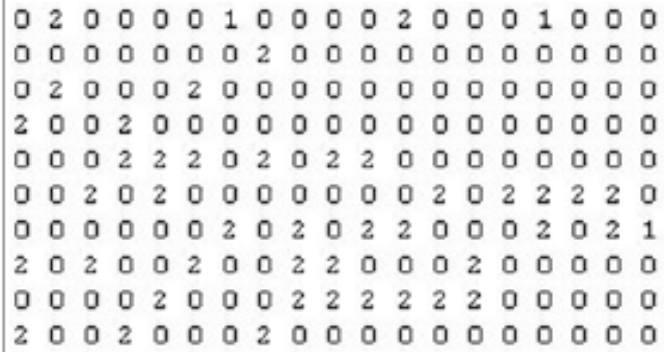

Fig 10. Arquivo ProfessorTurma

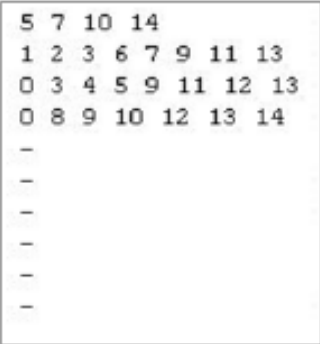

Fig 11. Arquivo RestriçãoHorárioTurma

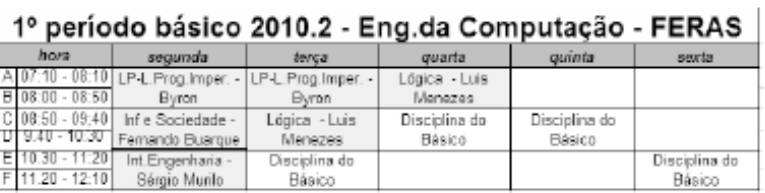

Fig 12. Resultado Grade Horária turma 0 ( $1^{\circ}$ periodo).

$2^{\circ}$ periodo básico 2010.2 manhã - Eng.da Computação

\begin{tabular}{|c|c|c|c|c|c|c|}
\hline & hars & sogunata & tanca & quarta & quinta & sexta \\
\hline 音 & $\frac{07: 10-08: 10}{08: 00-08: 50}$ & 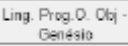 & $\begin{array}{l}\text { Desciplina do } \\
\text { Bassico }\end{array}$ & $\begin{array}{l}\text { Disciplina do } \\
\text { Básico }\end{array}$ & $\begin{array}{l}\text { Disciplina do } \\
\text { Elssico }\end{array}$ & \\
\hline$\frac{c}{D}$ & $\frac{08: 50-09 \cdot 40}{8.40-10: 30}$ & $\begin{array}{c}\text { Disciplina do } \\
\text { Basica }\end{array}$ & Ling Prog O Obj & Dise do Basico & & $\begin{array}{c}\text { Disciplina do } \\
\text { Basico }\end{array}$ \\
\hline $\mathrm{E}$ & $\begin{array}{l}10.30-11: 20 \\
11.20-12: 10 \\
\end{array}$ & $\begin{array}{l}\text { Disciplins do } \\
\text { Bdsico }\end{array}$ & & & $\begin{array}{l}\text { Disciplins do } \\
\text { Elssico }\end{array}$ & \\
\hline
\end{tabular}

Fig 13. Resultado Grade Horária turma 1 ( $2^{\circ}$ Período).

\begin{tabular}{|c|c|c|c|c|c|}
\hline \multicolumn{6}{|c|}{$3^{\circ}$ período básico 2010.2-manhã - Eng.da Computação } \\
\hline hora & segunda & tarca & quarta & quimte & sexta \\
\hline \begin{tabular}{|l|}
$07: 10-08.10$ \\
$08.00-08: 50$
\end{tabular} & $\begin{array}{l}\text { Disciplina do } \\
\text { Basico }\end{array}$ & $\begin{array}{c}\text { Disciplina do } \\
\text { Básico }\end{array}$ & Est Dacbs - Byron & $\begin{array}{l}\text { Discipina do } \\
\text { Bsasico }\end{array}$ & $\begin{array}{l}\text { Disciplina do } \\
\text { Easico }\end{array}$ \\
\hline \begin{tabular}{|l|}
$08.50-09 ; 40$ \\
$09.40-10: 30$ \\
\end{tabular} & Est Dsdas - Eyrom & & $\begin{array}{l}\text { Ag Aplic Comp - } \\
\text { Eliane Loiols }\end{array}$ & & $\begin{array}{l}\text { Diaciplina do } \\
\text { Basico }\end{array}$ \\
\hline \begin{tabular}{|l|}
$10.30-11: 20$ \\
$11.20-12: 10$ \\
\end{tabular} & $\begin{array}{l}\text { Alg Áplic Comp - } \\
\text { Elane Loings }\end{array}$ & $\begin{array}{c}\text { Diaciplina do } \\
\text { Bisico }\end{array}$ & & $\begin{array}{c}\text { Discipina do } \\
\text { Bisico } \\
\end{array}$ & \\
\hline
\end{tabular}

Fig 14. Resultado Grade Horária turma 2 ( $3^{\circ}$ Período).

\begin{tabular}{|c|c|c|c|c|c|}
\hline \multicolumn{6}{|c|}{$4^{\circ}$ período básico 2010.2 manhã - Eng.da Computação } \\
\hline han & segunda & terça & quarta & quinta & sexta \\
\hline 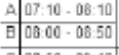 & $\begin{array}{c}\text { Disciplina da } \\
\text { Básico }\end{array}$ & \begin{tabular}{|l|} 
Sinsis c \\
- Danik
\end{tabular} & $\begin{array}{l}\text { L. Prog } \mathrm{F} \\
\text { Eruno Fe }\end{array}$ & $\begin{array}{r}\text { Discip } \\
\text { B4 }\end{array}$ & $\begin{array}{c}\text { Disciplina dr } \\
\text { Básico }\end{array}$ \\
\hline \begin{tabular}{l|l|} 
& $0850-09: 40$ \\
$D$ & $9.40-10: 30$ \\
\end{tabular} & $\begin{array}{c}\text { Sinais a Sistemas } \\
\text { Danid Chares }\end{array}$ & $\begin{array}{l}\text { L. Prog Funcio- } \\
\text { Bruno Femandes }\end{array}$ & & $\begin{array}{c}\text { Disciplina do } \\
\text { Bisica } \\
\end{array}$ & $\begin{array}{c}\text { Digciplina do } \\
\text { Básico }\end{array}$ \\
\hline \begin{tabular}{l|l|} 
& $10.30-11.20$ \\
$F$ & $11.20-12.10$ \\
\end{tabular} & & & $\begin{array}{l}\text { Discipina do } \\
\text { Aśsico }\end{array}$ & & $\begin{array}{c}\text { Disciplina do } \\
\text { Basico }\end{array}$ \\
\hline
\end{tabular}

Fig 15. Resultado Grade Horária turma 3 ( $4^{\circ}$ Periodo).

$5^{\circ}$ período 2010.2 manhă - Eng.da Computação

\begin{tabular}{|c|c|c|c|c|c|}
\hline horg & segunda & 105 & qubarta & quinta & sexta \\
\hline 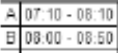 & $\begin{array}{c}\text { OreComb - Drriel } \\
\text { Chares }\end{array}$ & Redes 1-Edison & $\begin{array}{l}\text { Teomp - Eliane } \\
\text { Loiala }\end{array}$ & $\begin{array}{c}\text { Teamp- Elison } \\
\text { Laidala }\end{array}$ & \\
\hline \begin{tabular}{l|l} 
c & $00: 50 \cdot 09: 40$ \\
0 & $9,40 \cdot 10: 30$ \\
\end{tabular} & Redes 1 - Edsen & $\begin{array}{c}\text { CircCemb - Daniel } \\
\text { Chavas }\end{array}$ & $\begin{array}{l}\text { Mat Cierchica - } \\
\text { Simone Sartas }\end{array}$ & $\begin{array}{l}\text { Mast Ciemtnca - } \\
\text { Simone Santos } \\
\end{array}$ & \\
\hline \begin{tabular}{|l|l|}
$E$ & $10.30-11.20$ \\
$F$ & $11.20-12: 10$
\end{tabular} & Eng Seft - Genezio & $\begin{array}{l}\text { Eng Soft - } \\
\text { Cendzio }\end{array}$ & $\begin{array}{l}\text { Eletronica - Josá } \\
\text { Paulo }\end{array}$ & \begin{tabular}{|c|}
$\begin{array}{c}\text { Elatrorica - Joso } \\
\text { Poulo }\end{array}$ \\
\end{tabular} & \\
\hline
\end{tabular}

Fig 16. Resultado Grade Horária turma 4 ( $5^{\circ}$ Período). 


\begin{tabular}{|c|c|c|c|c|c|}
\hline \multicolumn{6}{|c|}{$6^{\circ}$ período 2010.2 manhã - Eng.da Computação } \\
\hline hara & seguads & tergat & Quarra & Quinca & \\
\hline \begin{tabular}{|l|l|}
$A$ & $07: 10-00: 10$ \\
$B$ & $0600-00 \cdot-50$ \\
\end{tabular} & $\begin{array}{l}\text { Sist Co } \\
\text { Camne }\end{array}$ & $\begin{array}{c}\mathrm{Ba} \\
\mathrm{Man}\end{array}$ & $\begin{array}{l}\text { Sist Com } \\
\text { Commel }\end{array}$ & & \\
\hline \begin{tabular}{|l|l|l|}
$C$ & $08.50-09: 40$ \\
$D$ & $9.40-10.30$ \\
\end{tabular} & $\begin{array}{l}\text { Ban } \\
\text { Masias }\end{array}$ & Redes 2- & $\begin{array}{c}\text { Cire Sequ - Sárgjo } \\
\text { Camp }\end{array}$ & $\begin{array}{l}\text { Ans: } \\
\text { Den }\end{array}$ & \\
\hline \begin{tabular}{|l|l|}
$E$ & $10: 30-11: 20$ \\
$F$ & $1120 \cdot 12: 10$
\end{tabular} & 2-Edison & Circ Saqu - S & \begin{tabular}{|c|} 
Ava Proj. Soff - \\
Denis Siveirs
\end{tabular} & & \\
\hline
\end{tabular}

Fig 17. Resultado Grade Horária turma 5 ( $6^{\circ}$ Período).

\begin{tabular}{|c|c|c|c|c|c|}
\hline \multicolumn{6}{|c|}{$7^{\circ}$ período 2010.2 manhä - Eng.da Computação } \\
\hline hord & sogunda & ferç̧a & quarta & quinta & sexta \\
\hline$\frac{07.10-08.10}{38.00-08.50}$ & $\begin{array}{l}\text { 14. Fennando } \\
\text { Evarnue }\end{array}$ & $\begin{array}{l}\text { AA - Femando } \\
\text { Quarquas }\end{array}$ & \begin{tabular}{|c} 
Cont Procesos- \\
Wellington -
\end{tabular} & $\begin{array}{l}\text { Mot Forngis - } \\
\text { Luis Menezes }\end{array}$ & $\begin{array}{c}\text { Cont Procesos } \\
\text { Welington }\end{array}$ \\
\hline $\begin{array}{l}08: 50 \cdot 09 ; 40 \\
9 \cdot 40 \cdot 10 \cdot 30\end{array}$ & $\begin{array}{l}\text { Compilad . } \\
\text { Gustaio Carv }\end{array}$ & $\begin{array}{l}\text { Compilad, - } \\
\text { Gustano Cans }\end{array}$ & $\begin{array}{l}\text { Mat Fomais - Luis } \\
\text { Menczas }\end{array}$ & $\begin{array}{l}\text { Avquit Corre - } \\
\text { Sergio Munilo }\end{array}$ & 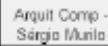 \\
\hline$\left|\frac{10.30-11.20}{11.20-12: 10}\right|$ & $\begin{array}{l}\text { Sist Operacional } \\
\text { Jose Puulo }\end{array}$ & $\begin{array}{l}\text { Sist Opsracion: } \\
\text { Jose Poulo }\end{array}$ & $\begin{array}{l}\text { Adm - Arany } \\
\text { Tirassugs }\end{array}$ & & \\
\hline
\end{tabular}

Fig 18. Resultado Grade Horária turma 6 ( $7^{\circ}$ Periodo).

$8^{\circ}$ período 2010.2 manhä - Eng. da Computaçäo

\begin{tabular}{|c|c|c|c|c|c|c|}
\hline han & segunds & terga & quarta & guînta & sexta & \\
\hline $\begin{array}{l}\text { A } 07 \cdot 10.08 \cdot 10 \\
B \quad 08: 00.0850 \\
\end{array}$ & $\begin{array}{c}\text { Proj gD - Elian } \\
\text { Laids }\end{array}$ & 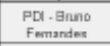 & $\begin{array}{c}\text { Sist Info- Simmono } \\
\text { Srrtas }\end{array}$ & $\begin{array}{c}\text { Sist Infh - Simane } \\
\text { Sintas }\end{array}$ & & \\
\hline \begin{tabular}{c|c|}
$\mathrm{C}$ & $(0.65-09.40$ \\
$\mathrm{D}$ & $9.40 \cdot 1030$
\end{tabular} & $\begin{array}{l}\text { Comunic Dighal1. } \\
\text { Cameis Heates }\end{array}$ & $\begin{array}{l}\text { Min de dados. } \\
\text { Meuservalenca }\end{array}$ & $\begin{array}{l}\text { POI- Erumo } \\
\text { Farrandese }\end{array}$ & $\begin{array}{c}\text { Proi E0 - Elisene } \\
\text { Loinla }\end{array}$ & & $\begin{array}{c}\text { Fom it Enverent } \\
\text { Enswallets }\end{array}$ \\
\hline $\begin{array}{l}E 10.30-11.20 \\
F \mid 11.20-12.10\end{array}$ & $\begin{array}{l}\text { Proj Camp. } \\
\text { Gustron Conn }\end{array}$ & $\begin{array}{l}\text { Proj Comp. } \\
\text { Gustson Com }\end{array}$ & \begin{tabular}{|c} 
Comunit Digitai1. \\
Camies Esetos
\end{tabular} & \begin{tabular}{|c|} 
Min de dados. \\
Meuter Volentras
\end{tabular} & & inents \\
\hline
\end{tabular}

Fig 19. Resultado Grade Horária turma 7 ( $8^{\circ}$ Período). Aulas em amarelo não foram contempladas pelo programa.

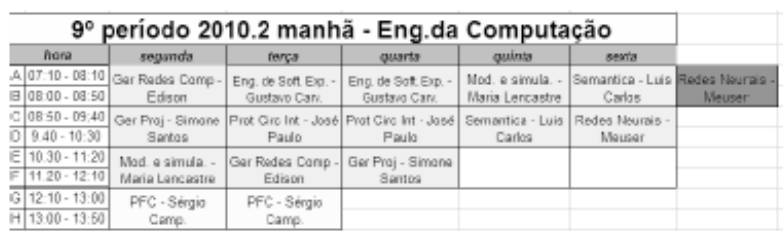

Fig 20. Resultado Grade Horária turma 8 ( $9^{\circ}$ Periodo). Aula em vermelho não recebeu horário viável.

\begin{tabular}{|c|c|c|c|c|c|}
\hline \multicolumn{6}{|c|}{$10^{\circ}$ período 2010.2 manhä - Eng.da Computação } \\
\hline hord & segunda & teryaga & quararta & govinta & sexta \\
\hline $\begin{array}{ll}07: 10-0610 \\
08.00-0650\end{array}$ & & $\begin{array}{l}\text { Afl Eng de Sct - } \\
\text { Cenezio - }\end{array}$ & $\begin{array}{l}\text { Ifícrocantol: } \\
\text { Danial Chaves }\end{array}$ & & \\
\hline $\begin{array}{ll}08: 50-0940 \\
940-10.30 \\
\end{array}$ & $\begin{array}{l}\text { Apl Eng de Son - } \\
\text { Genazio }\end{array}$ & & & & \\
\hline$\frac{10.30-11.20}{11.20-1210}$ & $\begin{array}{l}\text { Mcrocontrol. } \\
\text { Danisl Chasusa }\end{array}$ & $\begin{array}{l}\text { Visso Comput - } \\
\text { Enno Famandos }\end{array}$ & $\begin{array}{l}\text { Masác Compuc. } \\
\text { Bruno Fomandes }\end{array}$ & & \\
\hline
\end{tabular}

Fig 21. Resultado Grade Horária turma 9 ( $10^{\circ}$ Período).

\begin{tabular}{|c|c|c|c|c|c|}
\hline \multicolumn{6}{|c|}{ Bruno Fernandes } \\
\hline hora & segunds & ferces & Quarta & Quiata & sexte \\
\hline \begin{tabular}{|l|l|} 
A & $07: 10-00: 10$ \\
& $08: 00-08: 50$
\end{tabular} & & $\begin{array}{l}\text { PDI - Bruna } \\
\text { Famandes }\end{array}$ & $\begin{array}{l}\text { L. Prog Funcio- } \\
\text { Buna Fornandes }\end{array}$ & & \\
\hline \begin{tabular}{|l|l|} 
C & $06.50-09.40$ \\
$D$ & 940.10 .30
\end{tabular} & & $\begin{array}{l}\text { L. Prog Funcio- } \\
\text { Enno Femandes }\end{array}$ & $\begin{array}{l}\text { PDI. Erumo } \\
\text { Femandes }\end{array}$ & & \\
\hline 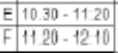 & & $\begin{array}{l}\text { Visäo Comput. } \\
\text { Bruno Fermand. }\end{array}$ & $\begin{array}{l}\text { Visso Comput. - } \\
\text { Bruno Fernand }\end{array}$ & & \\
\hline
\end{tabular}

Fig 22. Resultado Grade Horária professor 0 (Bruno Fernandes).

\begin{tabular}{|c|c|c|c|c|c|}
\hline \multicolumn{6}{|c|}{ Byron } \\
\hline mard & sogunda & forca & quanta & quints & sertia \\
\hline$\frac{\mathrm{A}}{\mathrm{B} 07-10 \cdot 08 \cdot 10}$ & $\begin{array}{l}\text { LP. Prog Imper. } \\
\text { Byran }\end{array}$ & $\begin{array}{l}\text { LP. L Prog irper } \\
\text { Byron }\end{array}$ & Eat Dadas - Byran & & \\
\hline 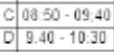 & Est Dad05 - Eyror & & & & \\
\hline $\begin{array}{c}E 10.30 \cdot 11: 20 \\
F 1120 \cdot 12 \cdot 10\end{array}$ & & & & & \\
\hline
\end{tabular}

Fig 23. Resultado Grade Horária professor 1 (Byron).

\begin{tabular}{|c|c|c|c|c|c|}
\hline \multicolumn{6}{|c|}{ Carmelo Bastos } \\
\hline in & Begunds & rearcs & Quasts & Quintras & serts \\
\hline $\begin{array}{l}A 07: 10-08 \cdot 10 \\
-06: 00-08: 50\end{array}$ & $\begin{array}{l}\text { GistComunicaras - } \\
\text { Camnelo }\end{array}$ & & $\begin{array}{l}\text { Sist Comuricasaso } \\
\text { Carmelo }\end{array}$ & & \\
\hline 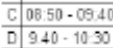 & 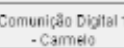 & & & & \\
\hline $\begin{array}{l}\mathrm{E} \mid \mathrm{E} \cdot \mathrm{.30}-11.20 \\
\mathrm{~F} / 11.20-1210\end{array}$ & & & 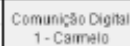 & & \\
\hline
\end{tabular}

Fig 24. Resultado Grade Horária professor 2 (Carmelo Bastos).

\begin{tabular}{|c|c|c|c|c|c|}
\hline \multicolumn{6}{|c|}{ Daniel Chaves } \\
\hline hara & segunds & tercas & Quarra & Quinca & sexte \\
\hline \begin{tabular}{|l|l|}
$\mathrm{A}$ & $07.10-08.10$ \\
8 & $08.00-08.50$ \\
\end{tabular} & $\begin{array}{c}\text { CireCamb - Danial } \\
\text { Chares }\end{array}$ & \begin{tabular}{|l} 
Shais e Sietemes \\
- Darial Chaves
\end{tabular} & $\begin{array}{l}\text { Mcrocentrol. } \\
\text { Danial Chaves }\end{array}$ & & \\
\hline \begin{tabular}{l|l|} 
C & $06.50-09.40$ \\
0 & $0.40-10.30$
\end{tabular} & $\begin{array}{c}\text { Sinais e Sistemas } \\
\text { DanielChariag }\end{array}$ & $\begin{array}{c}\text { CircConb - Daniel } \\
\text { Chases }\end{array}$ & & & \\
\hline \begin{tabular}{|l|l|}
$E$ & $10: 30-11: 20$ \\
$F$ & $1120-12: 10$ \\
\end{tabular} & $\begin{array}{l}\text { Micrecantrol. - } \\
\text { Dariel Chaves }\end{array}$ & \begin{tabular}{|l} 
Sinais e Sistamas \\
- Dariel Chavos
\end{tabular} & & & \\
\hline
\end{tabular}

Fig 25. Resultado Grade Horária professor 3 (Daniel Chaves).

\begin{tabular}{|c|c|c|c|c|c|}
\hline \multicolumn{6}{|c|}{ Edison } \\
\hline bara & stgundz & terso & quant & quints & $\operatorname{sex} 19$ \\
\hline \begin{tabular}{|l|l|} 
A & $07.10-08.10$ \\
$B$ & $08.00-08.50$ \\
\end{tabular} & $\begin{array}{c}\text { Ger Redes Camp - } \\
\text { Edison }\end{array}$ & Redes 1 - Edison & & Redes 1-Edison & \\
\hline \begin{tabular}{|l|l|}
$0090-09.40$ \\
0 & $9.40-10.30$
\end{tabular} & Radas 1-Ediaon & Redas 2 - Edison & & Rodos 2 - Edison & \\
\hline \begin{tabular}{|l|l|}
$E$ & $10: 30-11: 20$ \\
$F$ & $11.20-12: 10$ \\
\end{tabular} & Redes 2-Edison & $\begin{array}{c}\text { Ger Redes Cumi } \\
\text { Edison }\end{array}$ & & $\begin{array}{c}\text { Ger Redes Comp } \\
\text { Edison }\end{array}$ & \\
\hline
\end{tabular}

Fig 26. Resultado Grade Horária professor 4 (Edison).

\begin{tabular}{|c|c|c|c|c|c|}
\hline \multicolumn{6}{|c|}{ Eliane Loiola } \\
\hline hara & sequnds & tercya & quarta & quints & sextas \\
\hline $\begin{array}{l}\mathrm{A} \mid 07-10-08-10 \\
\mathrm{~B} 0300-08.50\end{array}$ & $\begin{array}{l}\text { Proj BD - Eliane } \\
\text { Loiola }\end{array}$ & & $\begin{array}{l}\text { Tcomp - Elane } \\
\text { Loidla }\end{array}$ & $\begin{array}{l}\text { Tcamp - Elisne } \\
\text { Loiols }\end{array}$ & \\
\hline$\frac{\mathrm{C} .0950 \cdot 09 \cdot 40}{0.90-10: 30}$ & & & Ang Aplis Comp - & Proj BD - Eliane & \\
\hline $\begin{array}{l}\text { E } 1030 \cdot 11: 20 \\
\text { F } 1120 \cdot 12.10 \\
\end{array}$ & $\begin{array}{l}\text { Alg Apic Comp. } \\
\text { Eliana Loiola }\end{array}$ & & & & \\
\hline
\end{tabular}

Fig 27. Resultado Grade Horária professor 5 (Eliane Loiola).

\begin{tabular}{|c|c|c|c|c|c|}
\hline \multicolumn{6}{|c|}{ Fernando Buarque } \\
\hline Bora & sogunda & ters & quartia & quinta & soxta \\
\hline 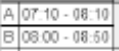 & $\begin{array}{l}\text { AA. Femando } \\
\text { Burque }\end{array}$ & $\begin{array}{l}\text { 1A - Fernendo } \\
\text { Buarque }\end{array}$ & & & \\
\hline \begin{tabular}{l|l|} 
C & $06.50-09 \cdot 40$ \\
0 & $0.40-10.30$
\end{tabular} & $\begin{array}{l}\text { Info Sociodsde- } \\
\text { Fermando Buarcue }\end{array}$ & & & & \\
\hline \begin{tabular}{l|l|}
$E$ & $10.30 \cdot 11: 20$ \\
$F$ & $11.20 \cdot 12: 10$
\end{tabular} & & & & & \\
\hline
\end{tabular}

Fig 28. Resultado Grade Horária professor 6 (Fernando Buarque).

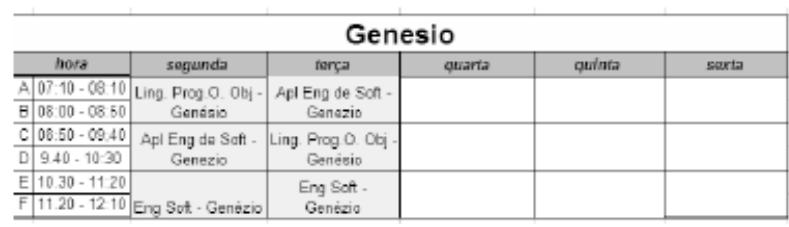

Fig 29. Resultado Grade Horária professor 7 (Genésio).

\begin{tabular}{|c|c|c|c|c|c|}
\hline \multicolumn{6}{|c|}{ Gustavo Carvalho } \\
\hline mard & sagundz & força & quaria & quints & searta \\
\hline 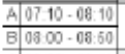 & & $\begin{array}{l}\text { Eng de Son Exp } \\
\text { Quatavo Cary }\end{array}$ & $\begin{array}{l}\text { Eng de Sof. Exp } \\
\text { Gustano Canv }\end{array}$ & & \\
\hline \begin{tabular}{l|l|} 
C $00.50-09,40$ \\
0 & $9.40-10.30$ \\
\end{tabular} & $\begin{array}{l}\text { Compiladores } \\
\text { Qust to Garaho }\end{array}$ & \begin{tabular}{|c|c|} 
Compiladores- \\
Gustana Carvalha \\
\end{tabular} & & & \\
\hline 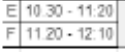 & $\begin{array}{l}\text { Proi Comp. } \\
\text { Gustaic Con }\end{array}$ & $\begin{array}{l}\text { Proj Comp. } \\
\text { Gustro Carv. }\end{array}$ & & & \\
\hline
\end{tabular}

Fig 30. Resultado Grade Horária professor 8 (Gustavo Carvalho). 


\begin{tabular}{|c|c|c|c|c|c|}
\hline \multicolumn{6}{|c|}{ Simone } \\
\hline hora & stgunds & terç & quarra & Quinta & $\operatorname{sen} 19$ \\
\hline \begin{tabular}{|l|l|}
$A 07: 10-00: 10$ \\
$B 09: 00-08: 50$ \\
\end{tabular} & & & $\begin{array}{c}\text { Sist Inta - Simons } \\
\text { Santua }\end{array}$ & $\begin{array}{c}\text { Sist into - Simono } \\
\text { Santos }\end{array}$ & \\
\hline \begin{tabular}{c|c|} 
C & $08.50-09,40$ \\
$D$ & $9.40-10: 30$
\end{tabular} & $\begin{array}{c}\text { Ger Proj - Simnene } \\
\text { Samtos }\end{array}$ & & $\begin{array}{l}\text { Met Cientifica - } \\
\text { Simone Sentos }\end{array}$ & \begin{tabular}{|l|} 
Met Cientifica - \\
Simone Sertios
\end{tabular} & \\
\hline \begin{tabular}{|l|l|} 
E $10.30-11.20$ \\
$F$ & $11.20-12: 10$ \\
\end{tabular} & & & $\begin{array}{l}\text { Gor Proj - Simone } \\
\text { Santua }\end{array}$ & & \\
\hline
\end{tabular}

Fig 31. Resultado Grade Horária professor 9 (Simone).

\begin{tabular}{|c|c|c|c|c|c|}
\hline \multicolumn{6}{|c|}{ José Paulo } \\
\hline hora & segende & fercs & quarts & quints & sexta \\
\hline $\begin{array}{l}\text { A } 07.10-08.10 \\
B \\
B\end{array}$ & & & & & \\
\hline$\frac{\mathrm{C} \cdot 08 \cdot 50 \cdot 09 \cdot 40}{\mathrm{D} 9.40 \cdot 10.30}$ & & $\begin{array}{l}\text { Prot Cinc Int - Jose } \\
\text { Paulo }\end{array}$ & $\begin{array}{l}\text { Proc Cire Im - Jose } \\
\text { Pado }\end{array}$ & & \\
\hline 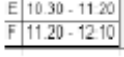 & $\begin{array}{l}\text { Sist Operacions } \\
\text { Jast Prulo }\end{array}$ & \begin{tabular}{|c} 
Sist Operacional . \\
Jose Paula
\end{tabular} & $\begin{array}{c}\text { Eletrorica - José } \\
\text { Pado }\end{array}$ & $\begin{array}{c}\text { Eletroniza - Jase } \\
\text { Pado }\end{array}$ & \\
\hline
\end{tabular}

Fig 32. Resultado Grade Horária professor 10 (José Paulo).

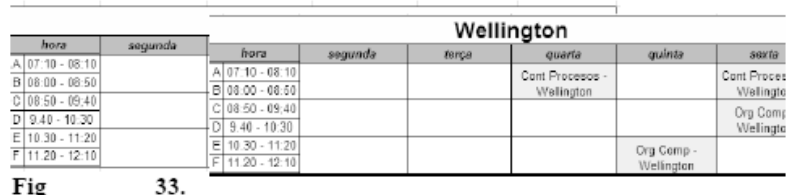

Resultado Grade Horária professor 11 (Luis Carlos).

\begin{tabular}{|c|c|c|c|c|c|}
\hline \multicolumn{6}{|c|}{ Maria } \\
\hline hora & Begunds & ferres & Qtesrms & quintas & sents \\
\hline $\begin{array}{l}A 07: 10-0810 \\
\theta 08: 00.08: 50\end{array}$ & & $\begin{array}{l}\text { Banc Dados. } \\
\text { Maria Lencestie }\end{array}$ & & $\begin{array}{l}\text { Modo a simula. } \\
\text { Maria Lencastre }\end{array}$ & \\
\hline 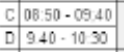 & $\begin{array}{l}\text { Banc Dados- } \\
\text { Marii Lençastie }\end{array}$ & & & & \\
\hline \begin{tabular}{l|l|}
$E$ & $10.30-11 \cdot 20$ \\
$F$ & $11.20-1210$
\end{tabular} & $\begin{array}{l}\text { Mod a simula. } \\
\text { Maria Lencestrit }\end{array}$ & & & & \\
\hline
\end{tabular}

Fig 34. Resultado Grade Horária professor 12 (Maria Lencastre).

\begin{tabular}{|c|c|c|c|c|c|c|}
\hline \multicolumn{6}{|c|}{ Meuser } & \\
\hline hag & segundi & resca & quariu & quinta & suria & \\
\hline$\frac{A}{807.10-0810}$ & & & & & & 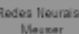 \\
\hline 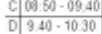 & & 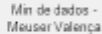 & & 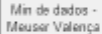 & 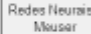 & \\
\hline 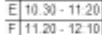 & & & & & & \\
\hline
\end{tabular}

Fig 35. Resultado Grade Horária professor 13 (Mêuser). Aula em vermelho não recebeu horário viável.

\begin{tabular}{|c|c|c|c|c|c|}
\hline \multicolumn{6}{|c|}{ Sérgio Campello } \\
\hline hara & segunds & renzas & quars & quints & sencts \\
\hline 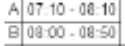 & & & & & \\
\hline 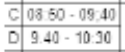 & & & $\begin{array}{l}\text { Circ Sequ - Särgio } \\
\text { Camp }\end{array}$ & & \\
\hline \begin{tabular}{|l|l|} 
E $10,30 \cdot 11: 20$ \\
$F$ & $1120 \cdot 12: 10$ \\
\end{tabular} & & $\begin{array}{c}\text { Cirr Saçu - Särgio } \\
\text { Camp }\end{array}$ & & & \\
\hline & $\begin{array}{c}\text { FFC-Serigio } \\
\text { Camp }\end{array}$ & $\begin{array}{l}\text { PFC - Sirgo } \\
\text { Camp. }\end{array}$ & & & \\
\hline
\end{tabular}

Fig 36. Resultado Grade Horária professor 14 (Sérgio Campello). Aulas em amarelo não foram contempladas pelo programa.
Fig 37. Resultado Grade Horária professor 15 (Sérgio Murilo)

\begin{tabular}{|c|c|c|c|c|c|}
\hline \multicolumn{6}{|c|}{ Denis } \\
\hline hord & segyenda & $2 \sec 8$ & quarte & quinta & $\operatorname{ser} 29$ \\
\hline \begin{tabular}{l|l|l} 
& $07: 10-08.10$ \\
\end{tabular} & & & & & \\
\hline \begin{tabular}{l|l|} 
& $08.50-09.40$ \\
0 & $9.40-10.30$
\end{tabular} & & & & $\begin{array}{l}\text { Ana. Proj Son - } \\
\text { Denis Shrera }\end{array}$ & \\
\hline \begin{tabular}{l|l}
$\mathrm{E}$ & $10.30-11 \cdot 20$ \\
$\mathrm{~F}$ & $11.20-12 \cdot 10$
\end{tabular} & & & $\begin{array}{l}\text { Ana Proj Sof - } \\
\text { Derig St }\end{array}$ & & \\
\hline
\end{tabular}

Fig 38. Resultado Grade Horária professor 16 (Denis).

Fig 39. Resultado Grade Horária professor 17 (Wellington).

\begin{tabular}{|c|c|c|c|c|c|}
\hline \multicolumn{6}{|c|}{ Jurany Travassos } \\
\hline hara & esgunds & terces & quara & quínta & $\operatorname{senta}$ \\
\hline 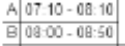 & & & & & \\
\hline 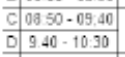 & & & & & \\
\hline 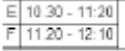 & & & $\begin{array}{l}\text { Admiristrachas . } \\
\text { Jurany }\end{array}$ & & \\
\hline
\end{tabular}

Fig 40. Resultado Grade Horária professor 18 (Jurany Travassos).

As aulas em amarelo não foram alocadas (já estavam presentes no mesmo horário e dia na gradehorária de 2010.2). Uma aula do Professor Mêuser da disciplina de Mineração de Dados não pôde ser alocada com as restrições vigentes.

\section{Conclusão}

Nota-se que existe uma relação entre o timetabling problem e o problema de coloração de grafos. Segundo Neufeld et al. (1974) a coloração do grafo gerará soluções possíveis, mas nem sempre viáveis. Por isso que essa coloração será apenas uma etapa de todo um processo para a geração de eficientes soluções para o problema.

A Seção 3 apresenta o procedimento criado por Souza et al. (2000) que visa a melhoria de soluções já existentes. Ele está presente na heurística descrita em Souza et al. (2001), mas poderia também melhorar resultados obtidos pela coloração de grafos de Neufeld et al. (1974). Souza et al. (2000) mostra também a importância de um estudo mais aprofundado de Souza et al. (2001) e de outras heurísticas existentes.

O programa feito foi limitado, pois gerou apenas uma solução inicial, porém gera soluções que atendem restrições básicas e pode com melhorias futuras gerar uma grade de horário eficiente. 


\section{Agradecimentos}

Ao PIBIC-POLI pela concessão da bolsa.

\section{Referências}

[1] BEASLEY D. Why Evolutionary Computation, in Bäck, T., Fogel, D. B. \& Michalewicz, Z. Handbook of Evolutionary Computation, Oxford University Press, 1997.

[2] CHU, S. C; FANG, H. L. Genetic Algorithms vs. Tabu Search in Timetable Scheduling.In: KNOWLEDGE-BASED INTELLIGENT INFORMATION ENGINEERING SYSTEMS, Third International Conference, Adelaide, SA, Australia, 1999, pp. 492-495.

[3] EVEN, S., Itai, A. E., Shamir, A. (1976). On the complexity of timetabling and multicommodity flow problems. SIAM Journal of Computing, 5:691-703.

[4] FRIEDMANN, C.V.P., Markenzon, L., Waga, C.F.E. In: SIMPÓSIO BRASILEIRO DE PESQUISA OPERACIONAL, 42., 2010. Rio Grande do Sul. Algoritmos Polinomiais para Colorações Ótimas em Algumas Famílias de Grafos. Saúde Humana, Saúde Animal e Ecossistema. São Paulo.

[5] KOFFMAN E. B; WOLFGANG P. A. T. Objetos, Abstração, Estrutura de Dados e Projeto Usando JAVA 5.0. 1st ed. LTC. 2008. 708p.

[6] NEUFELD, G. A; TARTAR J. Graph Coloring Conditions for the Existence of Solutions to the Timetable Problem. Communications of the ACM, v. 17, n. 8, p. 450-453,Agosto, 1974.

[7] SCHAERF, A., A Survey of Automated Timetabling, Artificial Intelligence Review, No. 13, 1999, pp. 87-127..

[8] SILVA, D. J; SILVA G. C. In: SIMPÓSIO BRASILEIRO DE PESQUISA OPERACIONAL, 42., 2010. Rio Grande do Sul. Heurísticas Baseadas no Algoritmo de Coloração de Grafos para o Problema de Alocação de Salas em uma Instituição de Ensino Superior. Saúde Humana, Saúde Animal e Ecossistema. São Paulo

[9] SOUZA, M. J. F; MACULAN N; OCHI L.S. Melhorando Quadros de Horário de Escolas através de Caminhos Mínimos. Tendências em Matemática Aplicada e Computacional, v. 1, n. 2, p. 515524, 2000.

[10] SOUZA, M. J. F; MACULAN N; OCHI L.S. Uma Heurística para o Problema de Programação de Horários em Escolas. Tendências em Matemática Aplicada e Computacional, v. 2,n. 1, p. 213222, 2001.

[11] VIANA, G. V. R. Meta-heurísticas para Solução de Problemas de Otimização Combinatória. 1996. 214 f. Dissertação (Mestrado) - Universidade Federal do Ceará, Fortaleza. 